\title{
Experiments with Multinational Cross-Course Project
}

\author{
Mehul S Raval \\ School of Engineering and Applied Science \\ Ahmedabad University \\ Ahmedabad, India \\ mehul.raval@ahduni.edu.in \\ Mazad Zaveri \\ School of Technology \\ Pandit Deendayal Petroleum University \\ Gandhinagar, India \\ mazad.zaveri@sot.pdpu.ac.in
}

\author{
Tolga Kaya \\ School of Computer Science and Engineering \\ Sacred Heart University \\ Fairfield, CT, USA \\ kayat@sacredheart.edu \\ Paawan Sharma \\ School of Technology \\ Pandit Deendayal Petroleum University \\ Gandhinagar, India \\ paawan.sharma@sot.pdpu.ac.in
}

\begin{abstract}
A modern engineer needs global competencies to work in a multinational team. Active learning during education helps to understand the nuances of such future engagements. Therefore, this paper discusses the dynamics of the crosscourse project for sophomores in a multicultural environment. The project is embedded within courses offered at two universities, which are spatially and temporally separated. At each university the course uses blended learning with project providing opportunities for online interactions and rest content coverage through classroom dialogues. The paper outlines the experiences of the students, faculty and provides recommendations for future implementations. The essential advisory is for: (1) Fine-tuning the framework for improving teamwork. (2) Supervising the groups and evaluating them as per the onshore university norms.
\end{abstract}

Keywords-active learning, competencies, cross-course project, internationalisation

\section{INTRODUCTION}

The advances in Information and Communication Technology (ICT) have accelerated the integration of world economies. It provides an opportunity for modern engineers to be a part of the global work market. Multinational companies often have campuses across the globe and projects in different countries. Students equipped with global competencies have better chances of employability [1], [2]. Top global competencies include the following [1] abilities: appreciate and communicate across cultures; work in a culturally and ethnically diverse team; deal with ethical issues arising due to cultural or national differences; developing practical solutions or products under the influence of cultural diversity. The development uses the well-known project-based learning (PBL) model [3].

There are several initiatives by governments that inculcate competencies and improve employment opportunities. For example, the ERASMUS + is an EU program that provides opportunities for students [4], National Student Exchange [5], or Idea League [6]. The ERASMUS + EPIC is a program focusing on employability with collaboration across industry and academia [7]. It promotes PBL and uses an international team of students to solve real-world problems. It promotes the use of ICT tools and open-source educational resources, making projects scalable and sustainable. Another European initiative ERASMUS+ COLIBRI [8], is implementing innovative teaching pedagogies using projects with student teams from different universities. It had a three-year cycle that uses blended learning activities involving 2 to 5 teachers and 3 to
5 students. The project proves that small multinational projects are beneficial for the students. The Technical University of Munich took up another student project initiative through a Global-Drive project [8], initiated by the Lehrstuhl für Fahrzeugtechnik (FTM). The project ran from 2008 - 09, and each year 3 to 4 FTM students worked on a project relevant to the automotive industry with a foreign team.

Many researchers use cross-course projects as a model for active learning [9]. The key idea is to share projects across two complementary courses in the semester. It enhances the project's scope, allows multiple faculty to interact, and students develop domain and life skills [10]. A cross-course collaborative PBL has shown improvement in student retention and confidence [10]. Another attempt at cross-course projects uses data management concentrations [11]. The advanced database design course project aligns with the industrial project in a course at higher level. The evaluation makes a positive case for integration based pedagogy as it improves student performance. Another attempt on a cross-course project, pair students from different technology-oriented software development courses [12]. The paper discusses problems encountered, faculty experiences, and recommendations to apply cross-course PBL.

In another multinational project execution, students spent a summer break at a host university undergoing PBL [13], [14]. The program [14] showed improvement in five competencies: hands-on skills, domain knowledge, critical thinking, intrinsic motivation, and teamwork. It also prepared students for the global labor market. Many times if the physical visit to campus is not feasible, then projects execute virtually using ICT. The challenges for a virtual environment interactions are [15]: the resolution of technical challenges, consistency, transparency in information flow, fairness in the problem statements; establishment of appropriate assessment measurements; realistic project definitions. The virtual interactions for project learning also impact socio-cultural aspects. These aspects include differences in languages, time management, attitude towards grades in the course; a different level of autonomy; assumptions about other cultures; work culture. Taiwan and Kansai University develop a global PBL curriculum and learning environment [16]. It employs PBL in a global setup to understand the socio-cultural aspects and uses IT (Information Technology) to generate virtual environments. The teams' spatial and temporal differences are solved by IT tools [16], [17] and cloud services. 
Thus it is seen that the projects for improving competencies have the following aspects: They are strategically funded by the governments [4]-[8] or driven by the university [9] in the specific area. It involves the industrial partner to support the project; a cross-course project involves complementary courses from the same university or organization [10]-[12]. Such experiences usually target senior year courses or capstone projects [11][12]; a student team may visit the host university under International exposure programs [13]. This engagement needs physical travel and stay for the duration of typically 4 to 12 weeks; the projects execute virtually using ICT [14][17] for students of diverse courses.

Unlike the above approaches [10]-[17], the proposed approach has the following characteristics:

- The project executes in a resource-constrained environment.

- It is a cross-course project, but it spans multiuniversity across the globe, unlike [10]-[12].

- The experiment covers semester III (sophomore - fall) courses (unlike seniors in [11]-[12]) to start early building of competencies.

- It spans similar courses, unlike [14]-[17], which had different course participation.

- The approach does not alter existing regulations at the onshore university.

Blended learning [19] combines online communication with offline classroom interactions to improve the learning experience. The paper uses blended learning with ICT tools facilitating dialogues between the teams and inputs from the offshore university instructor. The classroom dialogues cover the course's non-project content and bridge the gaps in students' understanding. The offline sessions also helped to chip off students' misgivings arising out of the online session interactions.

This paper's structure is as follows: an overview of the project setup and its organization is in Section II, the evaluation in Section III, the discussions and recommendations towards these experiments in Section IV, and the conclusion in Section V.

\section{Project Setup}

The project aims to provide an experience of developing technical skills while communicating and working in culturally diverse international team. The project definition considers: sophomore students without the advanced level technical knowledge, financial and infrastructural resource constraints, use of offshore university student-evaluation norms, availability of similar courses at both the university, and agreement among the faculty member for the experiment.

The instructors from Pandit Deendayal Petroleum University (PDPU), India, and Sacred Heart University (SHU), USA, shared their first project ideas about the implementation of Finite State Machine (FSM) for: (a) garden sprinkler system; (b) 7-Segment display, and counter; (c) the automatic car - throttle and gear-ratio control; (d) A signal strength indicator. After several discussions over email, the instructors from PDPU and SHU decided on the project (d).
The factors considered in deciding the project were: (a) understanding essential content necessary for its completion, (b) feasibility of project completion concerning timeline and duration, and (c) availability of software tools.

\section{A. Project Definition}

Students worked on implementing the Moore Finite State Machine for a signal strength indicator (an example shown in Fig. 1. The FSM implementation utilizes a freeware software, Logisim.

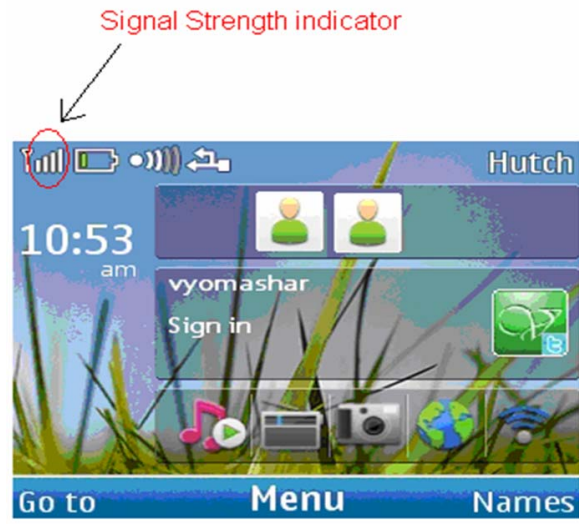

Fig. 1. Example of signal strength indicator bars.

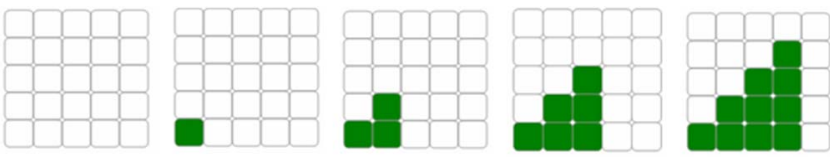

Fig. 2. Signal strength indicator bars for strength in the range 60 to 80 .

\begin{tabular}{|c|c|c|c|c|c|}
\hline & $\begin{array}{l}\dot{2} \\
\vdots \\
z \\
z\end{array}$ & $\begin{array}{l}\dot{z} \\
\dot{z} \\
\pm \\
\dot{0} \\
\pm \\
\beth\end{array}$ & $\begin{array}{l}z \\
2 \\
\infty \\
\infty \\
0 \\
\infty \\
n\end{array}$ & 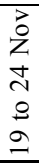 & 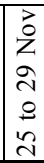 \\
\hline Announcement of project by email & & & & & \\
\hline $\begin{array}{l}\text { Create the state diagram for the FSM } \\
\text { Students can initially start thinking about the state } \\
\text { diagram individually, but as it evolves, the partners should } \\
\text { interact atleast twice over voice/video chat }\end{array}$ & & & & & \\
\hline $\begin{array}{l}\text { Each student will discuss implementation methods of } \\
\text { FSM (implementation in Logisim tool) with his/her } \\
\text { partner. } \\
\text { Distribute the work/methods, and individually implement } \\
\text { the assigned work. }\end{array}$ & & & & & \\
\hline $\begin{array}{l}\text { Each student will learn from, and verify the } \\
\text { implementations done by the partner. } \\
\text { Prepare individual reports/demo on the project. }\end{array}$ & & & & & \\
\hline Presentation/Demo to the corresponding instructors & & & & & \\
\hline
\end{tabular}

Fig. 3. GANTT chart showing the four weeks project timeline.

Depending on the signal strength (an 8-bit input of the FSM; valid range 0 to 100), the FSM would continuously display on a $5 \times 5$ LED matrix, the correct sequence of bars. For example, if the signal strength is between 60 to 80 , the FSM will generate the outputs (on a $5 \times 5$ LED matrix) that repeats, as illustrated in Fig. 2.

\section{B. Team Formation}

The course on Digital Design at SHU had eight students (7 students and one teaching assistant). At PDPU, the Digital Logic and Design course consisted of 147 students, of which eight students had to be selected to create eight pairs (one student from the PDPU and one student from the SHU). The 
partner details were communicated to the respective students by the faculty team. The faculty shared the joint project's idea with the 147 students (however, the actual project title was not declared) and invited self-nominations. It turned out that only eight students from the class showed interest.

The primary reason behind the low percentage of selfnomination was supposedly the perception of additional time commitment. It was purely the students' perception (based on their discussions with their peers), and the instructors had never mentioned that the cross-course project would be more difficult or involved. This brings an exciting insight into the cultural aspect of the students. The lack of self-nomination shows: inhibition to work with an unknown person at a different place and time-zone; there is also a fear of failure; perception about an inability to communicate effectively from a student with vernacular language background. The state of Gujarat, in India, offers a medium of instruction as Gujarati or English in K-12.

\section{Project Timelines and Execution}

As shown in Fig. 3, the project spans three weeks. Students spent the first week on the initial design, and the second week focused on the implementation and later its reporting. In the design part of the project, students interact twice a week over the online platform. The pair decided the time of their communication based on time zone constraint. The partners discussed the digital design and chose the complementary aspect of the implementation. The individual piece development and integration will sharpen their technical skills. The student would also explain his / her design aspect to the counterpart then prepare the demonstration with the report. These interactions will develop soft skills.

\section{ANALYSIS AND RESULTS}

\section{A. Quantitative Analysis: Pre-project Survey}

A pre-project survey reveals an exciting insight into selected students' preparedness to join the cross-course project exercise. The pre-project survey covered the questions related to students' grades and comfort level to work with FSM, the software tool, collaborating with an unknown person, and working with a peer in a different country.

Fig. 4 reveals that all selected students at PDPU had excellent ("A") grade. This indicates that students have a high caliber, and it would improve project experience for the partner. However, it is important to get participation from all levels. This necessitates that students' recruitment process must be more rigorous in the future run of this experiment. The students have a good grasp of the design of FSM and the use of the tool. So they were technically well placed for the project execution within two weeks. However, the comfort level to collaborate with an unknown person has variations (excellent to uncomfortable). The comfort level also shows differences when they have to work with a peer from another country. It shows the mental inhibition of the students.

Fig. 5 reveals that SHU students have variations in students' grades. This shows heterogeneity in their scholastic level. The students were comfortable (at excellent to fair level) working with FSM and the tool. They were open to collaborating with an unknown partner in a different country (at an excellent to fair level). It shows their open mind about collaboration. It is different from the mindset shown by the Indian students, where some of them were uncomfortable.

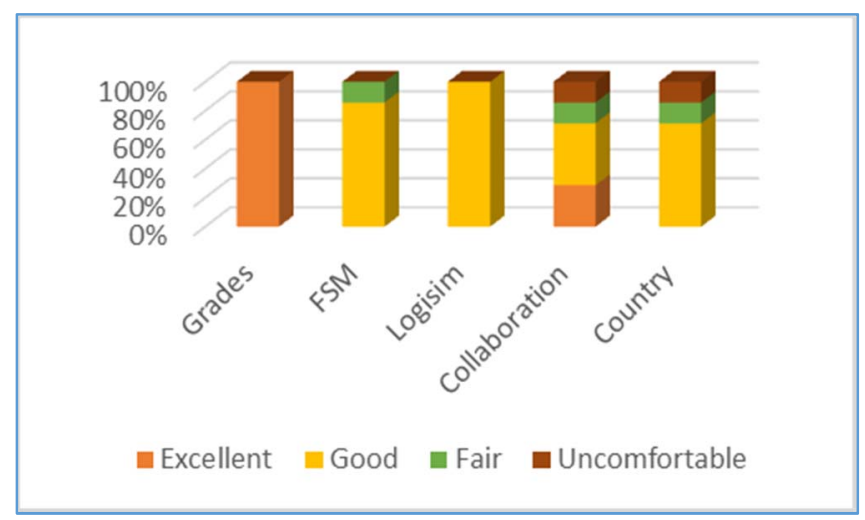

Fig. 4. Pre-project survey results for PDPU students.

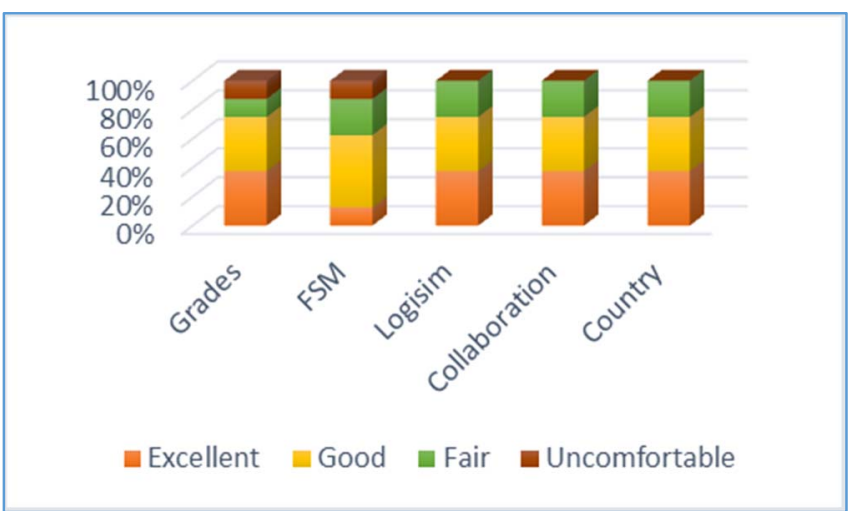

Fig. 5. Pre-project survey results for SHU students.

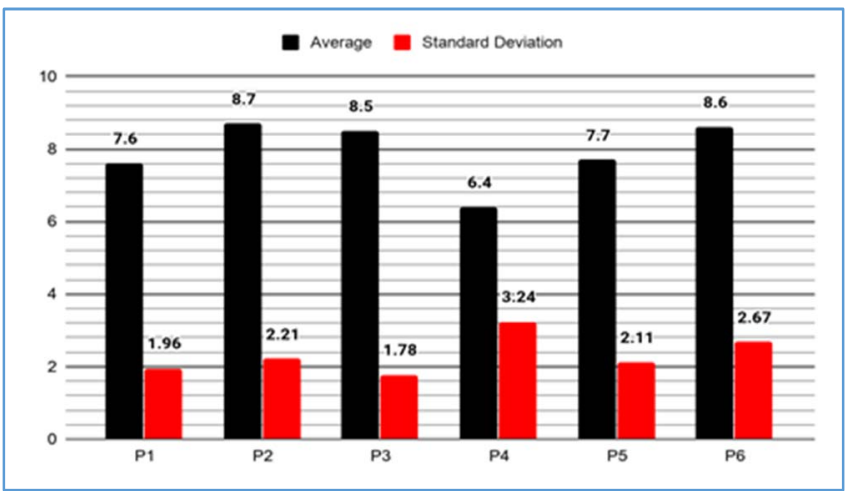

Fig. 6. MOS for the post-project survey for the PDPU students on scale 0 (unsatisfied) to 10 (fully satisfied).

\section{B. Post-project Survey}

Fig. 6 reflects the Mean Opinion Score (MOS) of the PDPU student's post-project satisfaction survey. The quantitative online survey questions are in Table I.

The first three-pointers were related to technical competency, and the next was related to life skills and overall satisfaction from the project. The online form also requested to provide qualitative comments on the following: (a) Describe the skills developed during the project; (b) Describe good points about this experiment of working with peers in other universities in a different country; (c) Suggest improvement in this experiment of working with peers in other universities in a different country. 
Fig. 6 reveals that PDPU students were satisfied with the technical competency development as reflected in the mean opinion score (MOS) of P2 and P3 for hands-on skills and knowledge improvement. The standard deviation (SD) is also reasonably low in these responses showing broad agreement. The P4 and P5 subsume the communication and teamwork aspects of the project. A high standard deviation in life skills means that opinion scores reflect a vast difference in experiences when working with colleagues across the university. The mean opinion score of P4 is low, with a very high standard deviation. The lower values reveal the glitches in communication as some students found communication difficult with the partner in other universities. It showcases an area where efforts are required to establish the synergy between the teams. It is also interesting to know that P5 has a relatively higher MOS and lower SD than P4. It is slightly puzzling as MOS for P4 and P5 provides different views in the students' thought processes. However, overall, students were eager to participate in the future runs of the projects. The post-project survey for the SHU students was qualitative, and Section IV covers it.

TABLE I. POST-PROJECT SURVEY QUESTIONS TO THE PDPU STUDENTS

\begin{tabular}{|l|l|l|}
\hline \multicolumn{1}{|c|}{ Competency } & Sr. No & \multicolumn{1}{|c|}{ Statement } \\
\hline \multirow{4}{*}{ Technical } & P1 & The project met my expectations. \\
\cline { 2 - 3 } Life & P2 & $\begin{array}{l}\text { I mark an improvement in hands-on } \\
\text { skills at the end of the project. }\end{array}$ \\
\cline { 2 - 3 } & P3 & $\begin{array}{l}\text { I have been able to improve my } \\
\text { knowledge of major at the end of the } \\
\text { project. }\end{array}$ \\
\hline P4 & $\begin{array}{l}\text { The other university colleague was } \\
\text { helpful in the project. }\end{array}$ \\
\cline { 2 - 3 } Overall & P5 & $\begin{array}{l}\text { Our team functioned well during the } \\
\text { project. }\end{array}$ \\
\hline P6 & $\begin{array}{l}\text { I would like to participate in similar } \\
\text { programs in the future. }\end{array}$ \\
\hline
\end{tabular}

TABLE II. Survey Questions TO THE FACUlty ABOUt THE CROSSCOURSE RROJECT

\begin{tabular}{|l|l|}
\hline Sr. No & \multicolumn{1}{c|}{ Statement } \\
\hline F1 & $\begin{array}{l}\text { The respective courses selected for the SHU and the PDPU } \\
\text { project match in coverage and depth. }\end{array}$ \\
\hline F2 & The project definition has good complexity. \\
\hline F3 & The project has a suitable time. \\
\hline F4 & $\begin{array}{l}\text { The project definitions executed at the P and the S have } \\
\text { complementary nature. }\end{array}$ \\
\hline F5 & The project is a valuable addition to the course. \\
\hline F6 & The students were excited to participate in this project. \\
\hline F7 & $\begin{array}{l}\text { The communication between faculty teams at S and P was } \\
\text { excellent. }\end{array}$ \\
\hline F8 & $\begin{array}{l}\text { The communication between the student team is smooth } \\
\text { during project execution. }\end{array}$ \\
\hline F9 & The evaluation mechanism is adequate for the project. \\
\hline F10 & $\begin{array}{l}\text { The experiment has value, and it should strengthen in the } \\
\text { future. }\end{array}$ \\
\hline
\end{tabular}

The Fig. 7 indicates the faculty perspective on the crosscourse projects. The survey pointers posed to the four faculty members involved in the project are shown in Table II. The online survey also requested faculty to provide qualitative comments to improve the design of the project.

Fig. 7 reveals that most mean opinion scores are high. The faculty felt that courses match well at both the Universities (F1). The project has suitable complexity (F2) and time frame (F3). The project adds value to the course (F5), and students are excited to participate in the project (F6), and the experiment should be strengthened in the future
(F10). The standard deviations for these MOS are also reasonably low, indicating consensus in the faculty views. The complementarity in the project (F4), communication amongst faculty teams (F7), and evaluation mechanism (F9) need fine-tuning. The standard deviation in these MOS is slightly high, and the faculty felt that these parts need attention. The lowest MOS concerns communication amongst the student teams (F8). The low SD indicates the uniformity in the thought process of faculty members. The faculty perspective also reveals gaps in communication between the students.

TABLE III. CORRELATION MATRIX (CAPTURING THE CORRELATION BETWEEN THE MARKS OBTAINED IN THE MID-SEMESTER EXAM, ENDSEMESTER EXAM, AND PROJECT)

\begin{tabular}{|c|l|l|l|}
\hline & End-Sem & \\
\hline End-Sem & 1.00 & Mid-Sem $^{\mathbf{a}}$ & Project $^{\mathbf{a}}$ \\
\hline Mid-Sem & 0.53 & 0.53 & 0.77 \\
\hline Project & 0.77 & 1.00 & 0.88 \\
\hline
\end{tabular}

TABLE IV. CORRELATION MATRIX (CAPTURING THE CORRELATION BETWEEN THE MARKS OBTAINED IN THE MID-SEMESTER EXAM, ENDSEMESTER EXAM, AND PROJECT)

\begin{tabular}{|c|l|l|l|}
\hline & \multicolumn{1}{|c|}{ End-Sem $^{\text {b }}$} & Mid-Sem $^{\text {b }}$ & Project $^{\text {b }}$ \\
\hline End-Sem & 1.00 & 0.56 & 0.99 \\
\hline Mid-Sem & 0.56 & 1.00 & 0.54 \\
\hline Project & 0.99 & $0.54 \quad{ }^{|c|}{ }^{\text {b. }}$ For the eight students at the SHU.
\end{tabular}

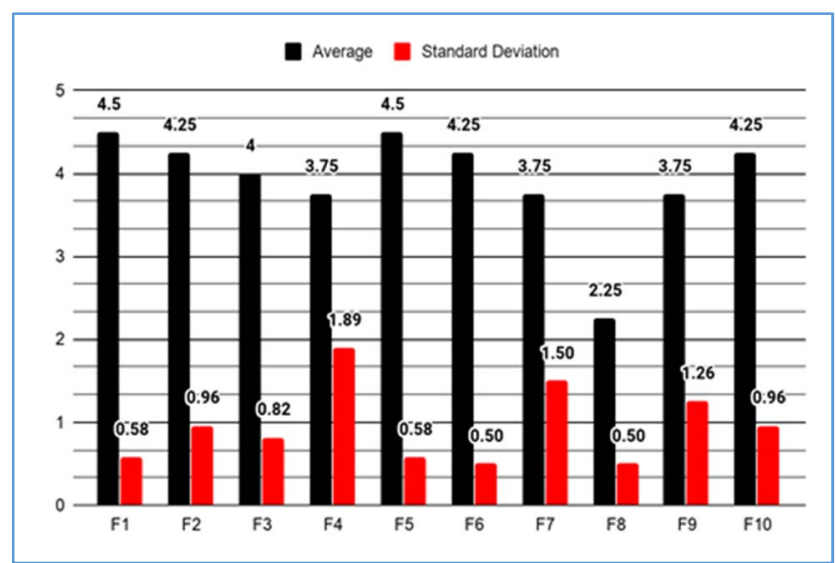

Fig. 7. MOS faculty survey about the project on scale 0 (Not satisfied) to 5 (fully satisfied).

The course runs in a blended learning mode. Therefore, it is crucial to evaluate the impact of learning gained through the project on the other course component. It is done by correlating the scores of the project with end semester exam scores of the students. Table III shows that the students' marks in the project are more correlated to the marks obtained in the end-semester exam. There is less correlation between the marks obtained in the project and the marks obtained in the mid-semester exam. The reason could be that the topics required for the project's implementation were covered mostly after the mid-semester exam.

The SHU students' correlation matrix calculated using the test 1 grades (Mid-Sem), project grades (homework 10\%, $15 \%$ of their grades), and the overall grades (End-Sem) is in Table IV. It can be seen that here also project grades were directly correlated with students' final grades. This is perhaps because the project assignment was towards the end of the semester. Mid-semester grades are not as correlated with the 
end of the semester, which can be attributed to the content being not internalized at the beginning of the semester.

Fig. 8 shows the final marks distribution of all the students of the Digital Logic and Design course at the PDPU. The numbers 2 and 6 written above the last two bins indicate students' numbers for the joint project. They were already outstanding in academics (high-performing students who score in the 80 to 100 marks range) and continue to perform well. Students reveal that during informal discussions, they did not perceive the joint project as an additional burden (in terms of time and effort).

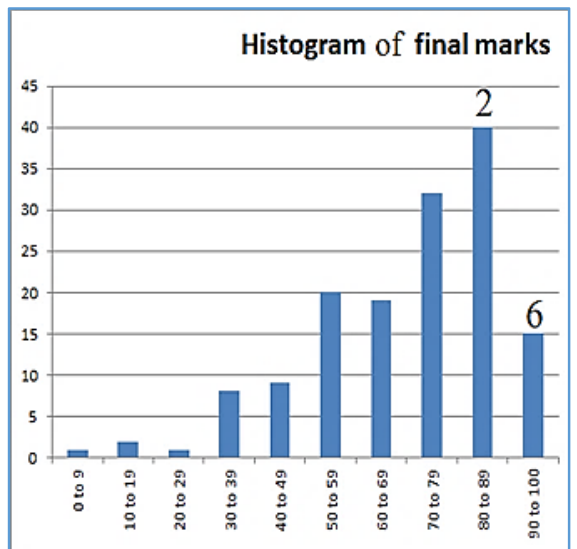

Fig. 8. Histogram of the final marks obtained by 147 students at the PDPU in the course.

\section{DISCUSSIONS}

\section{A. Qualitative Analysis: Pre-project}

The pre-project survey carried the following questions for the participants of both the university: (a) what do you think your biggest challenge will be in this project in terms of content? (b) What do you think your biggest challenge will be in this project in terms of collaboration (c) Why do you think this international collaboration is essential?

In response to above question (a) about the content, the PDPU students find FSM's implementation for the sudden change of signal strength or loss of signal strength very challenging. In response to question (b) related to collaboration, students felt challenges of the difference of time zone, communicating with the partner, difference of culture, and the prerequisite for the course, along with limitations of the tool. In response to the question (c), students were excited about learning new techniques from the partner, and its help in developing competencies, experience sharing to improve the learning and improve the relationship between the two universities.

\section{B. Qualitative Analysis: Post-project}

In general, all stakeholders: students, and instructors were satisfied with the outcomes of the experiments. The qualitative comments by the students provide insights into their thought process and team functioning. In response to questions about the experiments' plus points, students appreciated interactions with peers from other universities. They liked being out of their comfort zone and appreciating the different learning styles and approach to the problem. The students felt improvement in their communication skills and also technical and problem-solving skills. The students were excited about the implementations of FSM and mapping them to real-world problems.
The responses for improvement in the program suggests that communication is a challenge and more so due to time zone differences. There was some dissatisfaction with the lack of responses by the partner. The cooperation from peers could improve the overall functioning of the project. Some of the students also felt challenged in using the tool as all functionalities were not exact due to different versions. The student needed more time for the project and a prerequisite for the project be precise specified for the project's courses.

Some students provided informal feedback that one of the major hindrances in the cross-course project was the lack of mutual trust. The students were more focused on completing their academic tasks and would not be very open to collaboration with international students because of the short duration and the announcement towards the end of the semester. The above issues can be resolved by conducting ice breaking exercises before start of the semester.

The qualitative feedback from the faculty highlights the following points:

- Project planning can be done well in advance.

- How to make team members work with each other seamlessly in a coordinated manner? It appeared that the degree of association among the team members is low.

- The time zone difference between the two universities also hampers effective communication and a practical communication framework.

- Fine-tuning of the project definitions.

- Monitoring of teams during the execution of the project.

- Accommodating more teams for cross course project experience.

\section{Recommendations}

Based on quantitative and qualitative feedback, it is necessary to ensure effective working of the team. It is vital as the project involves: diverse cultures, different rules and regulations, language barriers, temporal and spatial differences, university traditions and precedence, and expectations set by the supervisors and the students. It makes it difficult to converge on the set of standard guidelines. Thus, such experiments are mostly successful with the involvement of the supervisors. The following recommendations may improve the future runs of the cross course projects.

- The faculty must clearly articulate the objectives and scope of the project to the students. They must identify and specify the role of the student. The clarity will help students see the advantages of the association and contribute without the fear of failure.

- The faculty must empower the students, and then jointly frame the learning objectives. The partnership by the students will improve their association and help students hone their skills.

- Faculty members of both universities should jointly supervise the team. However, it can be challenging to implement as the onshore university's faculty member may not whole heartedly support the project, influencing the participants. Therefore, all supervisors 
must also be motivated and know about the project's joint deliverables.

- The interactions among faculty members of both the universities must continue throughout the project duration.

- The documentation at every stage will go a long way in the success of the project. It should specify learning objectives, project outcomes, teams' tasks, and possibly workload per week. It must keep on evolving throughout project execution.

- In order to smoothen the regulations, the university may suggest a set of guidelines for such projects, e.g., joint report preparation.

- The recruitment process needs improvement to get participation from students at all academic levels.

\section{CONCLUSION}

The paper discusses the setup for international crosscourse projects. The objective is to provide the experience of working in a multicultural team to the students. The project spans a similar courses across the two universities in the early semester with a philosophy to "catch them young." It brings to fore challenges related to culture, language, rules and regulation, and time zone difference. The overall experience of the students and faculty is optimistic about the cross-course project experiments. However, there are areas of improvement, such as: teamwork, communication between teams, and joint-supervision. The paper gives various recommendations for addressing the above difficulties and improvising the future runs of the project. It highlights the needs that the project must continue to run in the future to clear out the aberrations observed during its first execution phase. The experiments have all the potential ingredients to motivate young kids and provide them with early cross-cultural experience.

\section{REFERENCES}

[1] K. Gardner, S. Stacy, and A. Walker, "Defining global competence for engineering students," in Amer. Soc. for Eng. Educ. Conf., 2011.

[2] L. Small, K. Shacklock, and T. Marchant, "Employability: A contemporary review for higher education stakeholders," J. Vocational Educ. and Training, vol. 70, no. 1, pp. 148-166, 2018.
[3] M. Raval, "Hybrid project based learning in computer vision," Int. J. Elect. Eng. and Educ., Sage Publishing, June, 2019.

[4] European Commission. (2020, May 28). "What is Erasmus+." [Online]. Available: https://ec.europa.eu/programmes/erasmusplus/about_en

[5] National Student Exchange. (2020, May 28). [Online]. Available: https://www.nse.org/

[6] Idea League. (2020, May 28). [Online]. Available: http://idealeague.org/

[7] EPIC. (2020, May 29). [Online] Available: http://epic.agu.edu.tr

[8] ERAMUS+ COLIBRI. (2020, May 28). [Online]. Available: https://www.tuhh.de/colibri/about/overview.html

[9] GlobalDrive. (2020, May 28). [Online]. Available: https://www.mw.tum.de/en/ftm/teaching/international-studentprojects/

[10] M. Raval, and R. Gandhi, "Experiments in active learning through project across courses," in IEEE Global Eng. Educ. Conf., April 2020, pp. 530- 539 .

[11] J. Winikus, L. Ziarek, C. Alphonce, and J. Hartloff, "Improving retention and confidence through cross-course collaborative projectbased learning," in IEEE Frontiers Educ. Conf., 2018, pp. 1-5.

[12] S. Mithun, and L. Xiao, "Incorporate cross-course knowledge integration into computing education," in IEEE Frontiers Educ. Conf., 2019, pp. 1-8.

[13] Nwokeji, C. Joshua, and P. S. T. Frezza, "Cross-course project-based learning in requirements engineering: An eight-year retrospective," in IEEE Frontiers Educ. Conf., 2017, pp. 1-9.

[14] M. Raval, and T. Kaya, "Effect of multinational projects on engineering students through a summer exposure research program," in IEEE Global Eng. Educ. Conf., April 2020, pp. 51-55.

[15] Brewer, P. Estes, A. Mitchell, R. Sanders, P. Wallace, and D. D. Wood, "Teaching and learning in cross-disciplinary virtual teams," IEEE Trans. Prof. Commun., vol. 58, no. 2, pp. 208-229, 2015.

[16] R. Hoda, M. Babar, Y. Shastri, and H. Yaqoob, "Socio-cultural challenges in global software engineering education." IEEE Trans. Edu., vol. 60, no. 3, pp. 173-182, 2016.

[17] T. Yamamoto, A. Liao, W. V. Wu, M. Shih, J. Shih, and H. Chu, "A proposal for the global and collaborative PBL learning environment where all global members on different campuses are - on the same page throughout the process of learning in the project," in IEEE Conf. Technologies and Applications in Artificial Intell., pp. 90-95, 2018

[18] R. Reis, C. Duque, S. Isotani, C. L. Rodriguez, K. T. Lyra, P. A. Jaques, and I. I. Bittencourt, "Affective states in computer-supported collaborative learning: Studying the past to drive the future," Comp. and Educ., vol. 120, pp. 29-50, 2018.

[19] C. Bonk, and C. Graham, "Blended learning systems," in The Handbook of Blended Learning: Global perspectives, Local designs, Wiley, 2006, pp. 3-21. 